\title{
Avoidance learning by squirrel monkeys: Measures and motivators'
}

\author{
V. J. POLIDORA, UNIVERSITY OF WISCONSIN \\ WILLIAM N. BOYER, OKLAHOMA CITY UNIVERSITY
}

Air-blast US led to more rapid acquisition of consistent shuttle avoidance behavior in squirrel monkeys than did continuous or discontinuous electric grid-shock US. The noise of the air-blast alone did not elicit consistent avoidance or escape behavior. A measure of the development of consistent behavior, trials to successively more stringent runs criteria, was shown to reveal group differences that were obscured by the conventional plot of percentage avoidances as a function of trials.

Reasonably rapid and stable avoidance learning to electric grid-shock is difficult to obtain with unrestrained monkeys presumably because a monkey's unconditioned response to shock is so violent and chaotic. Recently, Rohles (1965) reported that two stump-tailed monkeys learned a Sidman-type schedule to avoid "winds" of different velocities and temperatures. Ray reported that rats $(1966 \mathrm{a}, \mathrm{b})$ and cats (1966c) rapidly learn a shuttle avoidance response to a buzzer CS followed by air-blast US. Although neither investigator directly compared air US with the conventional electric grid-shock US, their data suggest that learning is more rapid with an air US. We have directly compared the effectiveness of electric gridshock US with that of air-blast US in shuttle avoidance learning and extinction to a visual $C S$ in squirrel monkeys.

\section{Method}

Thirty-six adult squirrel monkeys (Saimiri sciureus) were randomly assigned to one of five groups (see below). None of the Ss had previously been shocked or otherwise punished in an experimental setting.

The shuttlebox was built of plywood walls (19 in. high), a grill floor, and transparent ceiling. The floor (12 in. wide by 27 in.) consisted of $20,3 / 4$ in.-wide, flat, stainless steel bars spaced $1 / 2$ in. apart. Being electrically isolated from one another and running the width of the box, these bars were used both for delivering shock and for detecting S's left-right position (via two contact relays). When continuous shock ( $2 \mathrm{ma}$ ) was delivered, S's position was recorded manually by $\mathrm{E}$ while he observed $\mathrm{S}$ via a TV monitoring system in use at all times. Discontinuous shock consisted of a 0.2 sec. shock once per sec.

Air-blast $\left(100 \mathrm{lb} / \mathrm{in}^{2}\right)$ was delivered through two 1/2-in. pipes positioned across the width of the box 1 in. from and parallel to both the grid floor and either end of the box. There were 150.043 in. holes spaced 1.5 in. apart in each pipe, 7 aimed parallel to the floor and 8 (staggered by $3 / 4$ in.) aimed $20^{\circ}$ from the horizontal. To deliver only the noise of the air-blast, the two pipes were rotated downward so that the air stream was directed out of the box. S entered the box through one of the two vertically sliding, translucent doors at either end of the box, and a $100 \mathrm{w}$ bulb (CS light) and reflector were mounted on the outside of each of these doors. A trial began when the CS light went on in the compartment occupied by $\mathrm{S}$; a shuttle response was recorded only when $\mathrm{S}$ entered the opposite compartment and left the original compartment.

On the first day of testing $S$ was first adapted to the box for $10 \mathrm{~min}$. and then presented with either 2 or 5 CS-only trials. No S shuttled rapidly and consistently in the CS-only trials, and when $S$ failed to shuttle after 10 sec., E terminated the CS. After the CS-only trials, each $S$ received 50 avoidance trials (VI.45 sec.). During each subsequent daily session (if any) a $10 \mathrm{~min}$. adaptation period preceded 50 avoidance trials, and each $S$ was tested until it reached the dual criterion of at least 18 avoidance responses on the last 20 trials and fewer than 400 ITI responses in the session (maximum of five sessions). ${ }^{2}$ Shuttle responses between trials (ITI responses) were without effect but were recorded. For the Air Noise Only group, E terminated the trial if $S$ failed to make an escape response after 5 sec. of US. Immediately after attaining criterion performance, each $S$ received consecutive daily extinction sessions, each consisting of $10 \mathrm{~min}$. adaptation followed by 50 extinction trials, until it met the criterion of 18 failures to avoid in the last 20 trials of a session (maximum of three sessions).

\section{Results and Discussion}

Escape latencies for the $20 \mathrm{Ss}$ in the Air-Blast groups were greater than 2 sec. on only six trials; practically all escape latencies were between 0.5 and 1.5 sec., even on the first trial. These data alone convincingly demonstrate the aversive properties of air-blast and support Ray's (1966c) observation that the directionality of air-blast predisposes $S$ to make rapid and efficient escape shuttle responses. Escape latencies for the Air Noise Only group were similarly short in the initial sessions but steadily increased thereafter; these Ss even failed to escape on about $40 \%$ of the trials in sessions 3-5.

Of more importance, however, are the avoidance learning data ${ }^{3}$ presented in Fig. 1. This conventional plot shows that air-blast US apparently produced shuttle avoidance learning at least as rapidly as did shock. The Air Noise Only group showed ostensibly 
similarly acquisition initially but more variable avoidance behavior in sessions 3-5. The extinction data showed complete extinction in two sessions for the Air-Blast groups, no extinction in three sessions (150 trials) for the Shock groups, and an erratic pattern for the Air Noise Only group.

The conventional plot of avoidance learning (e.g., Fig. 1) is a somewhat gross representation of acquisition data. This plot tends to obscure what can be considered a more important and experimentally significant attribute of acquisition-the rate of development of consistent behavior. In some cases trials to successively more stringent percentage criteria $(1 / 10$, $2 / 10, \ldots, 10 / 10)$ reveal subtle differences between groups (cf., Horel, Bettinger, Royce, \& Meyer, 1966). An even more discriminating analysis proved to be one in which trials to successive runs criteria were examined (i.e., trials required by each $S$ to reach the criteria of a run of one, then a run of two consecutive avoidances,..., through a run of 10 consecutive avoidance responses). All Ss in the Air-Blast and Shock groups attained these criteria.

The group medians of these data (Fig. 2) show striking differences between groups. ${ }^{3}$ The two AirBlast groups attained the criteria at a roughly linear rate and were not significantly 4 different from one another at any runs criterion. The two Shock groups also did not differ, ${ }^{4}$ but both required significantly 4 more trials than did either of the Air-Blast groups to reach each of the five longest runs criteria. The conclusion that the Air Noise Only group never did develop consistent avoidance behavior is obviously substantiated by the fact that this group did not even attain a run-of- 6 criterion within 250 trials.

The relative shapes of these curves (Fig. 2) are also revealing. Compared to the Air-Blast groups, the Shock groups attained the run-of-6 criterion at a much slower rate but subsequently developed con-

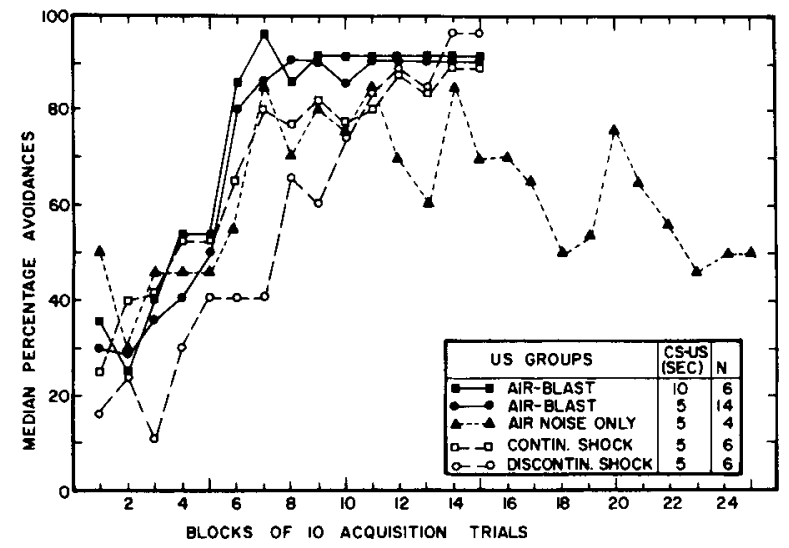

Fig. 1. Median percentage avoidance responses for the 5 groups in successive blocks of 10 trials.

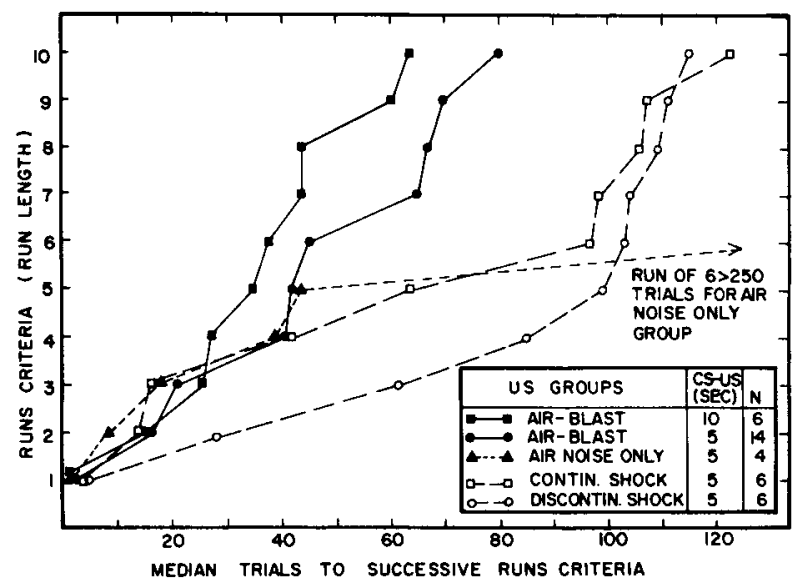

Fig, 2. Group medians of the number of trials required to attain successively more stringent runs criteria. Note that the independent variable, runs criteria, is plotted on the ordinate, and the dependent variable, trials to criteria, is on the abscissa.

sistent avoidance behavior at a highly similar rate. Thus these curves illustrate that the well-known disruptive effects of shock are most pronounced early in learning-having the effect of retarding the development of even moderately consistent avoidance behavior. Once $S$ has learned to avoid shock somewhat regularly, however, $\mathrm{S}$ seldom fails to avoid thereafter. Air-blast apparently produces more rapid acquisition simply by not retarding it.

\section{References}

Horel, J. A., Bettinger, L. A., Royce, G. J., \& Meyer, D. R. Role of neocortex in the learning and relearning of two visual habits by the rat. $J$. comp. physiol. Psychol., 1966, 61, 66-78.

Ray, A. J., Jr. Shuttle avoidance: Rapid acquisition by rats to a pressurized air unconditioned stimulus. Psychon. Sci., 1966a, $5,29-30$.

Ray, A. J., Jr. Nonincremental shuttle-avoidance acquisition to pressurized air US. Psychon. Sci., 1966b, 5, 433-434.

Ray, A. J., Jr. Shuttle avoidance: An apparatus for cats utilizing an air-blast unconditioned stimulus, $J$, genet. Psychol., $1966 \mathrm{c}$, 109,131 .

Rohles, F. H., Jr. Wind as an aversive stimulus. J. exp. Anal. Behav., 1965, 8, 203-205.

\section{Notes}

1. Supported in part by NIH grant FR-0167 and by Research Contract DA18-035-AMC-368(A). In conducting the research reported herein, the investigators adhered to the "Principles of Laboratory Animal Care" as established by the National Society for Medical Research. Assistance of D. A. Losey is gratefully acknowledged. 2. Two Ss in the Continuous Shock and $1 \mathrm{~S}$ in the Discontinuous Shock groups made an excessive number of ITI responses in the 1 st or 2nd session. Since deleting the data from these Ss did not materially change the group medians or statistical results, the data presented are based on all Ss tested.

3. Medians were more representative of each group's performance because $1 \mathrm{~S}$ in the Discontinuous Shock group learned very slowly; means were similar to medians for the remaining 4 groups.

4. Mann-Whitney U test, $\alpha=.01$. 